\title{
Percutaneous closure of paravalvular leak (fistula) between aorta and left atrium using echocardiographic guidance
}

\author{
Joanna M. Kirsch ${ }^{1}$, Tomasz Witkowski ${ }^{2}$, Marcin Protasiewicz ${ }^{2}$, Piotr Brzozowski ${ }^{1}$, Krzysztof Reczuch ${ }^{3}$, \\ Wiktor Kuliczkowski \\ ${ }^{1}$ Doctor Intern, Wroclaw University Hospital, Wroclaw, Poland \\ ${ }^{2}$ Department of Cardiology, Wroclaw Medical University, Wroclaw, Poland \\ ${ }^{3}$ Department of Heart Diseases, Wroclaw Medical University, Wroclaw, Poland
}

Adv Interv Cardiol 2021; 17, 3 (65): 317-318

DOI: https://doi.org/10.5114/aic.2021.109236

We present a case of a 67-year-old woman who was admitted to the hospital due to suspicion of a paravalvular leak around the artificial aortic valve. The patient had undergone the procedure of simultaneous implantation of aortic and mitral mechanical valves 8 years before the current problem: in both the aortic and the mitral position. Carbomedics valves were implanted in order to treat native valve stenosis. The patient complained of dyspnea, reduced physical exercise tolerance and atypical chest pain for 7 months. Transthoracic echocardiography revealed good function of the artificial valves with normal left ventricle ejection fraction, whereas transesophageal echocardiography (TEE) showed leakage (a fistula) in the anterior part of the aortic ring in the direction towards the left atrium. Neither computed tomography (CT) angiography nor coronarography showed evident features of the fistula. After consultation with the Heart Team, the patient was qualified for percutaneous closure of the fistula. The procedure was conducted through right femoral access. As we were not able to visualize the leak on fluoroscopy, the whole procedure was guided using TEE (Figure 1). The passage through the aortic paravalvular leakage towards the left atrium was obtained using the Sion guidewire. Then, using the telescopic technique a $5 \mathrm{~F}$ diagnostic catheter was introduced. After replacing the soft guidewire with a stiff one, an AR1 6F diagnostic catheter was used. Then, a $90 \mathrm{~cm}$ Cook $6 \mathrm{~F}$ vascular sheath was inserted into the left atrium. The attempt to close the leakage with a 1 Amplatzer Vascular Plug III (AVP III) $6 \times 3 \mathrm{~mm}$ failed, as even after positioning the device in the fistula significant leakage was present, despite the fact that previous measurements in the TEE suggested that 1 AVP III was sufficient. The leak was finally successfully closed with 2 AVP III $6 \times 3 \mathrm{~mm}$ devices. The second device was added sequentially, while first one was held on the delivery system. Both devices were then implanted simultaneously. The multiplug approach with AVP III devices is a well-documented technique for transcatheter paravalvular leak closure [1]. The patient was discharged in good overall condition 1 day after the procedure. A follow-up visit after 12 months confirmed good clinical and echocardiographic effects of the procedure. The patient did not complain of dyspnea, chest pain or loss of consciousness.

According to the literature, a fistula between the ascending aorta and the atrium is a rare condition [2]. The most common causes of this type of fistula are endocarditis and aortic aneurysms complicated with aortic dissection, but neither of these causes was present in our patient. This type of fistula is closed with an open surgical approach in almost $75 \%$ of cases. Percutaneous intervention is less frequent and is used in about $10 \%$ of cases [1], but as our case showed, it can still be an effective method of treatment. This case demonstrates that sometimes this type of leakage can only be seen in TEE so great caution is advised.

\section{Conflict of interest}

The authors declare no conflict of interest. 

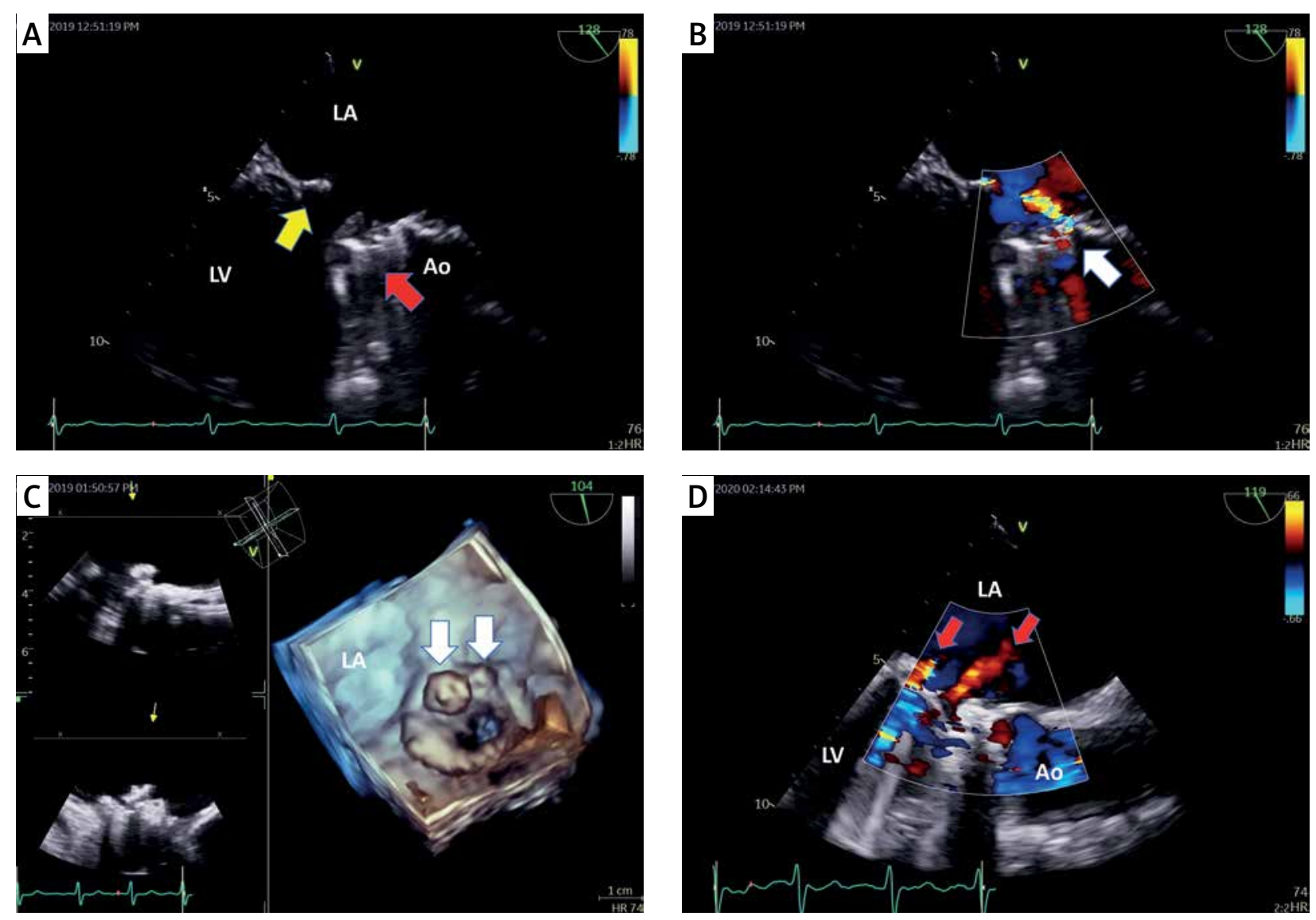

Figure 1. A - Transesophageal echocardiography, the mid-esophageal long-axis view $\left(120^{\circ}\right)$. The yellow arrow points to the prosthetic mechanical valve in the mitral position, the red arrow points to the prosthetic mechanical valve in the aortic position. B - The same image as in panel A with color Doppler imaging showing paravalvular leak originating from the aortic bulb and flowing into the left atrium (white arrow points to the origin of the jet in the aortic bulb). C - Transesophageal echocardiography. Three-dimensional reconstruction showing position of the implanted AVP III devices (white arrows). D - Transesophageal echocardiography, the mid-esophageal long-axis view $\left(120^{\circ}\right)$. Color Doppler imaging showing the absence of a previously present paravalvular leak. The red arrows point to the benign transprosthetic jets of the mechanical valve in the mitral position

$L A$ - left atrium, LV - left ventricle, Ao - aorta.

\section{References}

1. Smolka G, Pysz P, Jasiński M, et al. Multiplug paravalvular leak closure using Amplatzer Vascular Plugs III: a prospective registry. Catheter Cardiovasc Interv 2016; 87: 478-87.

2. Jainandunsing JS, Linnemann R, Bouma W, et al. Aorto-atrial fistula formation and closure: a systematic review. J Thorac Dis 2019; 11: 1031-46. 